\title{
BMJ Open Hemicraniectomy versus medical treatment with large MCA infarct: a review and meta-analysis
}

\author{
Paul Alexander, ${ }^{1}$ Diane Heels-Ansdell, ${ }^{2}$ Reed Siemieniuk, ${ }^{2,3}$ Neera Bhatnagar, ${ }^{4}$ \\ Yaping Chang, ${ }^{2}$ Yutong Fei, ${ }^{2,5}$ Yuqing Zhang, ${ }^{2}$ Shelley McLeod, ${ }^{6}$ \\ Kameshwar Prasad, ${ }^{7}$ Gordon Guyatt ${ }^{2}$
}

To cite: Alexander P, HeelsAnsdell D, Siemieniuk R, et al. Hemicraniectomy versus medical treatment with large MCA infarct: a review and meta-analysis. BMJ Open 2016;6:e014390. doi:10.1136/bmjopen-2016014390

- Prepublication history and additional material is available. To view please visit the journal (http://dx.doi.org/ 10.1136/bmjopen-2016014390).

Received 21 September 2016 Accepted 30 September 2016

CrossMark

For numbered affiliations see end of article.

Correspondence to Paul Alexander; elias98_99@yahoo.com

\section{ABSTRACT}

Objective: Large middle cerebral artery stroke (space-occupying middle-cerebral-artery (MCA) infarction (SO-MCAi)) results in a very high incidence of death and severe disability. Decompressive hemicraniectomy (DHC) for SO-MCAi results in large reductions in mortality; the level of function in the survivors, and implications, remain controversial. To address the controversy, we pooled available randomised controlled trials (RCTs) that examined the impact of DHC on survival and functional ability in patients with large SO-MCAi and cerebral oedema.

Methods: We searched MEDLINE, EMBASE and Cochrane library databases for randomised controlled trials (RCTs) enrolling patients suffering SO-MCAi comparing conservative management to $\mathrm{DHC}$ administered within 96 hours after stroke symptom onset. Outcomes were death and disability measured by the modified Rankin Scale (mRS). We used a random effects meta-analytical approach with subgroup analyses (time to treatment and age). We applied GRADE methods to rate quality/confidence/ certainty of evidence.

Results: 7 RCTs were eligible ( $n=338$ patients). We found DHC reduced death (69-30\% in medical vs surgical groups, $39 \%$ fewer), and increased the number of patients with $\mathrm{mRS}$ of 2-3 (slight to moderate disability: $14-27 \%$, increase of $13 \%$ ), those with mRS 4 (severe disability: 10-32\%, increase of 22\%) and those with mRS 5 (very severe disability $7-11 \%$ : increase of $4 \%$ ) (all differences $p<0.0001)$. We judged quality/confidence/certainty of evidence high for death, low for functional outcome mRS 0-3, and moderate for mRS $0-4$ (wide Cls and problems in concealment, blinding of outcome assessors and stopping early).

Conclusions: DHC in SO-MCAi results in large reductions in mortality. Most of those who would otherwise have died are left with severe or very severe disability: for example, inability to walk and a requirement for help with bodily needs, though uncertainty about the proportion with very severe, severe and moderate disability remains (low to moderate quality/confidence/certainty evidence).

\section{Strengths and limitations of this study}

- Inclusion of all published randomised trial data.

- Reproducible duplicate assessment of both eligibility and risk of bias.

- Appropriate sensitivity and subgroup analyses and, rating of the quality of evidence using the GRADE approach.

- Those of the primary studies, for example, risks of bias problems included lack of concealment of randomisation, lack of blinding of outcome assessors and stopping early because of large effects.

- Small sample sizes.

\section{BACKGROUND}

Large cerebral infarction is typically associated with devastating clinical outcomes, including severe neurological disability, brain herniation and death. ${ }^{1-8}$ Massive malignant middle-cerebral-artery (MCA) infarction (spaceoccupying MCA infarction (SO-MCAi)) is particularly devastating: cerebral oedema that occurs in the fixed intracranial space results in increasing intracranial pressure (ICP), increasing ischaemic cell death and in many instances leading to brain herniation and death. $^{7-13}$

Customary treatment for acute stroke and severe oedema is to reduce ICP using hyperosmotic agents, artificial ventilation and hyperventilation, therapeutic hypothermia, elevated head position and sedatives. ${ }^{14}$ Clinical trial evidence to support these strategies is, however, unavailable and they are at best modestly effective. ${ }^{14} 15$

Surgical decompression with hemicraniectomy and durotomy/duroplasty (external decompression involving removal of cranium overlying the oedematous brain tissue) is an aggressive approach that rapidly reduces 
ICP ${ }^{15} 16$ and thus may have a beneficial effect on neurological outcomes. ${ }^{8} 1617$ At the same time there are risks involved with hemicraniectomy including hydrocephalus, external brain tamponade, sinking skin flap syndrome, seizures, cerebral haemorrhage and paradoxical brain herniation. ${ }^{17}$ 18-20 More important, if hemicraniectomy reduces death but survivors suffer severe permanent disability, the value of the benefit may be questionable.

In this review we examined the effects of decompressive hemicraniectomy versus medical management (at times referred to as best management, standard care or conservative management) in patients suffering SO-MCAi with threatened brain oedema on mortality risk and disability at 6 months to 1 year. The included studies, and the essential conclusions, are similar to other recent systematic reviews of this question. ${ }^{21-23}$ This review is the first to use the GRADE approach to summarise the evidence, uses all available data and provides a schematic presentation (numbers and percentages at each Rankin Score cut-point) of results. The resulting perspective is likely to be particularly useful for clinicians in shared decision-making with patients' families.

\section{METHODS}

Eligible studies: (1) were RCTs (2) included patients suffering major stroke (MCA) with threatened brain oedema or evidence of increased intracranial pressure (3) assigned patients to either conservative or usual best medical practice (the control group) or hemicraniectomy (intervention group) within 96 hours after the onset of stroke symptoms and (4) reported at least death, or disability using the modified Rankin Scale (mRS), with follow-up of at least 6 months to 1 year (12 months; table 1$)$.

\begin{tabular}{ll} 
Table 1 & The modified Rankin Scale Rankin $^{24}$ \\
score & Description \\
\hline 0 & $\begin{array}{l}\text { No disability; no symptoms at all } \\
\text { No significant disability despite symptoms: } \\
\text { able to carry out all usual activities despite } \\
\text { symptoms }\end{array}$ \\
2 & $\begin{array}{l}\text { Slight disability: no assistance with one won } \\
\text { affairs but unable to carry out all previous } \\
\text { activities }\end{array}$ \\
3 & $\begin{array}{l}\text { Moderate disability: requiring some help, but } \\
\text { able to walk without assistance } \\
\text { Moderately severe disability: requiring } \\
\text { assistance to walk and to attend to own } \\
\text { bodily needs } \\
\text { Severe disability: bedridden, incontinent and } \\
\text { requiring constant nursing care and attention } \\
\text { Dead }\end{array}$ \\
\hline
\end{tabular}

\section{Search}

We accepted that a Cochrane review ${ }^{21}$ had conducted a comprehensive search up to October 2010. For our 2015 updated meta-analysis and electronic searching, we searched (1) MEDLINE (August 2010-January 2015) (2) EMBASE (August 2010-January 2015) (3) the Cochrane Database for Systematic Reviews (up to January 2015) and (4) Cochrane CENTRAL for clinical trials based on the search strategy in the prior Cochrane review. ${ }^{21}$ We enlisted the help of a medical librarian. We also searched the reference lists of all eligible articles or related reviews.

\section{Eligibility determination, risk of bias, data abstraction and quality of evidence assessment}

Following calibration exercises, reviewers, working independently in pairs, identified and retrieved the full texts of potentially eligible titles and abstracts. Subsequently, working independently and in pairs, reviewers made final adjudication of eligibility, judged risk of bias and abstracted data. For all eligibility determination, risk of bias assessment and data abstraction, reviewers resolved disagreement through discussion and, if necessary, third party adjudication. Reviewers used a modified Cochrane Risk of Bias Tool ${ }^{26}{ }^{27}$ using response options of 'yes', 'probably yes', 'probably no' and 'no'; the first two categories represented low risk of bias, and the latter two high risk of bias. This eliminated the often elevated 'unclear' response options.

We sought to collect data on a variety of trial characteristics and functional measures including the National Institutes of Health Stroke Scale, the Barthel index and the Hamilton Depression Rating Scale. The data proved, however, too incomplete to be informative. Therefore, we focused on the outcomes of death and disability measured by the modified mRS. ${ }^{24} 25$

We used the GRADE approach ${ }^{28-31}$ to rate the quality (certainty or confidence in effect estimates) of the body of evidence for death and disability. We considered issues of risk of bias (allocation concealment, blinding, incomplete data), consistency of study estimates (heterogeneity), directness (applicability of evidence to the study question), precision (95\% CIs) and publication bias, and summarised results in an evidence profile. ${ }^{31}$ We were prepared to assess the impact of loss to follow-up at the level of the entire body of evidence. ${ }^{32}$

\section{Analysis}

For eligibility decisions and for rating risk of bias we calculated chance-corrected agreement using $\kappa .{ }^{33}$ Studies measured outcome at several time points; we focused on and present data/analysis at 12 months. We built forest plots and conducted meta-analyses calculating the pooled relative treatment effects (relative risks (RR)) and associated 95\% CIs using random-effects inverse variance weighted modelling using thresholds of (1) dead or alive. (2) mRS of 3 or less versus $>3$ and (3) $\mathrm{mRS}$ of 4 or less versus $>4$. 
We measured heterogeneity using Cochrane- $\mathrm{Q}$ and $\mathrm{I}^{2}$ statistics and generated a priori hypotheses to explain heterogeneity including age of patients $(<60$ vs $>60$ years, anticipated benefit greater in those under 60 years) and timing of surgery (intervention $<48$ hours vs $\geq 48$ hours from symptom onset (up to 96 hours), anticipated benefit greater in earlier intervention). We used the $\chi^{2}$ test for subgroup differences to explore age and timing interactions for the outcome of mortality. Review Manager V.5.2.7 software was used to perform the meta-analyses. ${ }^{34}$

We calculated the total number and percentage of patients in the intervention and control groups who, at 12 months, were classified as mRS 1 and 2, 3, 4, 5 and 6. The Cochran-Mantel-Haenszel $\chi^{2}$ test for combining over multiple tables was used to test the differences in distributions. We modelled based on assumptions of ordinal and conducted a sensitivity analysis assuming non-ordinal data. We used the 6-month data which was the only data provided for one trial and include this $\left(\right.$ HeADDFIRST $^{35}$ ) and conducted a sensitivity analysis omitting these data.

\section{RESULTS}

Figure 1 presents the process by which we determined that, of the 1159 citations identified, seven ${ }^{17}{ }^{35-40}$ proved eligible for review inclusion (see online supplementary material file for an example of the MEDLINE search strategy). Agreement ( $\kappa)$ for the full title and abstract screening was 0.85 , and for the full text screening 0.76 . Inter-rater agreement on individual domains of the risk of bias tool ranged from 0.80 to 1.0 across the seven domains.

\section{Effects of interventions}

Table 2 presents trial characteristics. All included patients had suffered SO-MCAi and all included trials except for one ${ }^{40}$ were multicentre in design. Only seven patients were lost to follow-up ${ }^{17} 40$ and thus no adjustments for attrition ${ }^{32}$ were necessary. The seven trials that met the eligibility criteria were published from 2007 to 2014 and included 338 patients with 165 allocated to surgery group and 173 to medical management. The six trials that reported complete 12-month data involved 151 patients in the surgical group and 163 in the

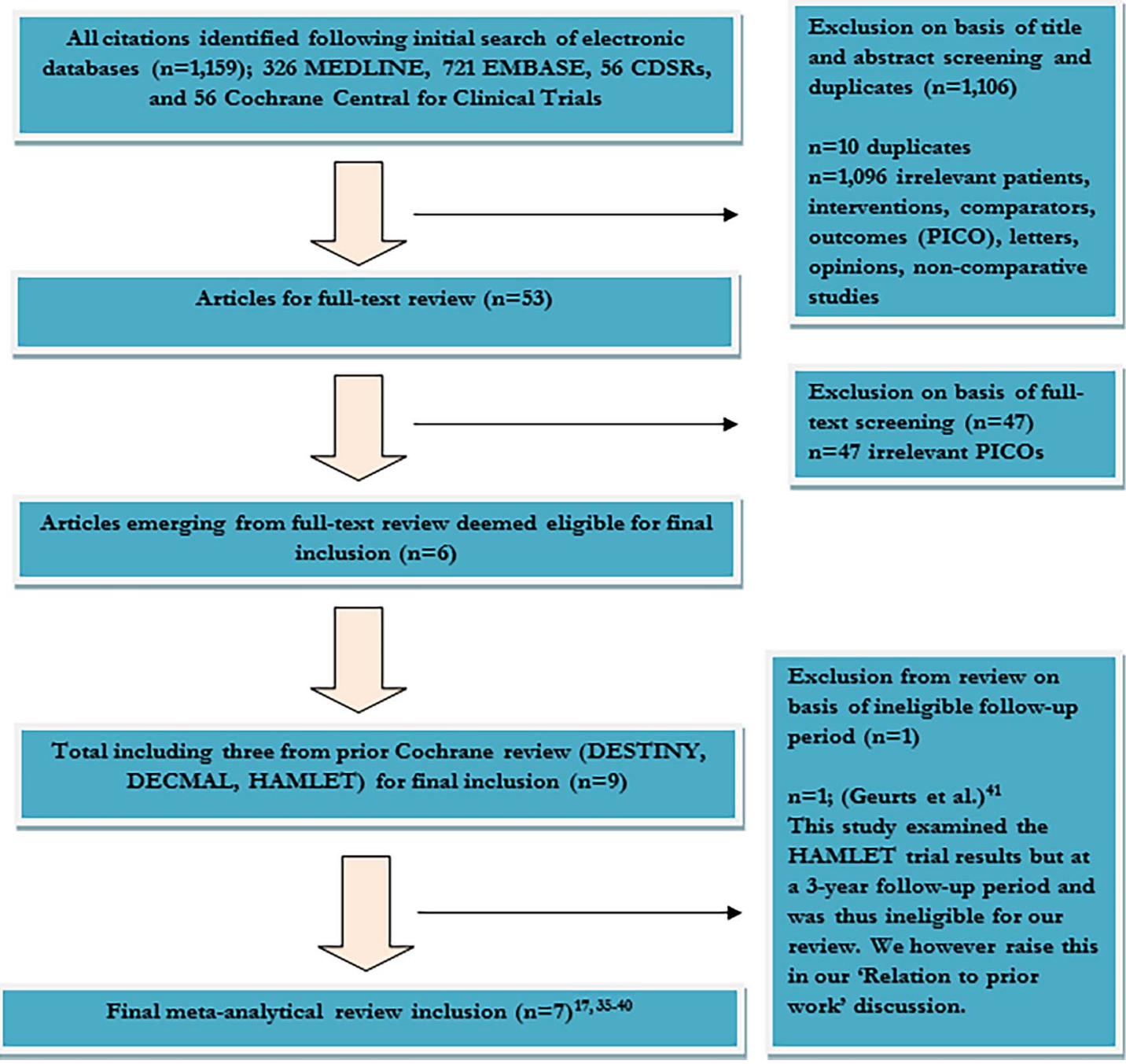

Figure 1 Flow diagram of summary of evidence searching and final RCT selection. RCT, randomised controlled trial. 
Table 2 Study characteristics

\section{Name, publication \\ year and reference \\ number, country, \\ first author \\ surname \\ Duration from symptoms onset \\ to treatment \\ Age (years) \\ inclusion; treatment}

Germany, Jüttler

after the onset of

symptoms

DESTINY I $2007,,^{38}>12$ to $<36$ hour Germany, Jüttler

DECIMAL 2007, ${ }^{37}$ France, Vahed

HAMLET 2009, 39 Netherlands,

Hofmeijer

HeADDFIRST 2014 Within 4 days pilot, ${ }^{35}$ USA and

Canada, Frank

(96 hours)

Within 4 days (96 hours)

Decompressive

Hemicraniectomy

2012, ${ }^{36}$ China,

Zhao

Decompressive

Hemicraniectomy

$2012,{ }^{40}$ Latvia,

Slezins

Surgery within

48 hours after

onset
Less than and

60 years; (61.5)

Over 60 years; $47 / 62 ; 50 \%$

Over 60 years; $47 / 62 ; 50 \%$ Anticipated sample size $\sim 130$ patients. Sequential analysis allowed for repeated interim analyses; trial stopped as soon as reached statistical significance for 'success' (proportion mRS 4 or less).

18-75 years;

$18-60$ years: 44.5

$18-55$ years; (43.4)

$18-60$ years; $(48.7)$

Surgery vs medical management (conservative treatment/ standard care)

Hemicraniectomy and duroplasty vs basic therapy in the ICU for stroke; osmotherapy with the use of mannitol, glycerol or

hypertonic hydroxyethyl starch; sedation; intubation and mechanical ventilation; hyperventilation; and administration of buffer solutions.

Hemicraniectomy plus conservative vs osmotherapy; intubation and mechanical ventilation; hyperventilation; venous

oxygenation; ICP monitoring; sedation; BP monitoring; head positioning; body core temperature; blood glucose level; fluid management; prophylaxis of DVT.

inclusion of 32 patients, the trial was interrupted according to the protocol because reached significance for the 30-day mortality end point.

Hemicraniectomy with or without duroplasty plus standard

20/18; 53\% Anticipated sample size of 60 patients; sequential analysis planned, stopped after the 38th patient due to slow recruitment, a large difference in mortality with ongoing European trials 3839

32/32; 41\% Planned sample size 112 , stopped early apparently because of large significant effect. angle of $30^{\circ}$; intravenous fluid restriction; intravenous mannito or furosemide; intravenous antihypertensive agents; prophylactic use of anticonvulsants.

Hemicraniectomy vs management in ICU consisting of osmotherapy with mannitol or glycerol; intubation and mechanical ventilation; hyperventilation; invasive monitoring of ICP; sedation; muscle relaxants; treatment of elevated BP; elevation of the head to an angle of $30^{\circ}$; maintenance of normothermia, normoglycaemia and normovolaemia. Hemicraniectomy and durotomy vs airway management; ventilator settings; BP control and agents; fluid and electrolyte management; gastrointestinal and nutritional management; haematological monitoring and management; ICP monitoring; sedation; use of mannitol; anticonvulsants; prophylaxis againstDVT; and rehabilitation. without further details.

18-80 years; $\quad 24 / 23 ; 28 \%$ Planned sample size was 110 ; trial was stopped after 47 patients recruited because of large, significant effect.

Hemicraniectomy plus duroplasty vs head positioning; osmotherapy; administration of intravenous mannitol or glycerol; fluid management; intravenous fluid restriction; pulmonary function and airway protection; intubation and mechanical ventilation; cardiac care; BP management; blood glucose management; sedation; no seizure prophylaxis; prevention of DVT and PE.

Hemicraniectomy plus best medical treatment group or the best medical treatment (BMT) alone group. No details were provided on the BMT approach.

$\mathrm{BP}$, blood pressure; DVT, deep-vein thrombosis; ICP, intracranial pressure; ICU, intensive care unit; PE, pulmonary embolism. 
medical group ( $\mathrm{n}=314$ ). Of the 338 patients, 134 participated in three trials ${ }^{37-39}$ that enrolled only patients under 60 years of age, 95 participated in three trials $^{35} 3640$ (one of which was based on 6-month data ${ }^{35}$ ) that enrolled patients both over and under 60 years, and 109 in one trial $^{17}$ that enrolled only patients over 60 years.

Figure 2 presents our assessment of risk of bias for the seven eligible studies. Important limitations include lack of concealment of randomisation in four studies, lack of blinding of outcome adjudicators in three studies, and stopping early because of large effects in five studies.

Figure 3A presents the observed distributions of Rankin scores in those patients who did and did not receive hemicraniectomy for all seven trials (including six trials with 12-month follow-up and one with 6-month follow-up data ( $\mathrm{p}$ for difference in distributions $<0.00001)$ ). Based on figure $3 \mathrm{~A}$, the hemicraniectomy group experienced $39 \%$ fewer deaths, $4 \%$ more patients in mRS category $5,22 \%$ more in category 4 , and $13 \%$ more in categories 3 or 2. Results were similar excluding patients with only 6-month follow-up. The distribution of disability and death was also similar in the five trials that provided 6-month data (figure 3B). The one trial that followed patients to 36 months ${ }^{41}$ suggested minimal differences in groups in those with mild to moderate disability.

Hemicraniectomy increased the likelihood of being a survivor (alive; figure 4) when compared with best medical treatment (RR 2.05, 95\% CI 1.54 to 2.72, $\mathrm{p}<0.00001, \mathrm{I}^{2}$ of $26 \%$ ) (high-quality evidence, table 3 ). Considering a mRS threshold of 3 or less versus 4 to 6 ,

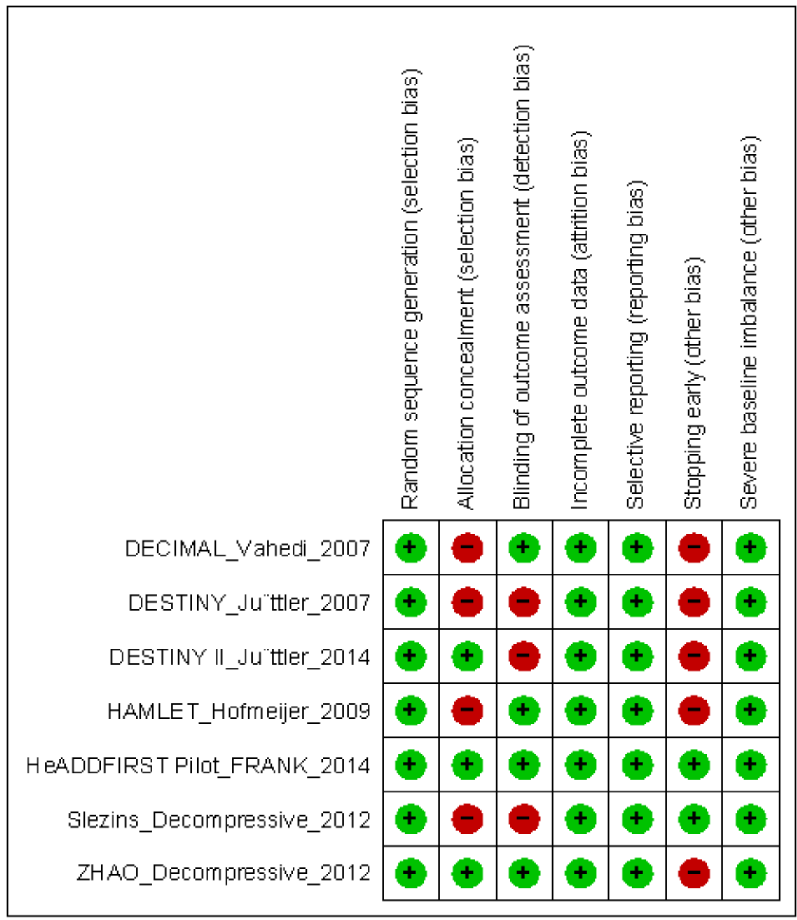

Figure 2 Risk of bias assessment. surgery increased the likelihood of being alive with an mRS of 3 or less (RR 1.58, 95\% CI 1.02 to 2.46, $\mathrm{p}=0.04$, $\mathrm{I}^{2}$ of $0 \%$, figure 5 , low-quality evidence, table 3 ). Considering a mRS threshold of 4 or less versus 5 or 6 (severe disability and death), surgery increased the likelihood of being alive and mRS 4 or less (RR 2.25, 95\% CI 1.51 to $3.35, \mathrm{p}<0.0001, \mathrm{I}^{2} 40 \%$, figure 6 , moderate quality evidence, table 3 ).

\section{Subgroup/sensitivity analyses}

The $\chi^{2}$ interaction test (test for subgroup differences) suggested similar effects in mortality for age $(\leq 60$ and $>60$ years old $)(p=0.38)$ and for duration between symptom onset and treatment initiation (up to 48 hours vs 96 hours $) \quad(p=0.59)$. Any differences could be explained by chance.

\section{DISCUSSION}

\section{Main findings}

Evidence from seven randomised trials ${ }^{17} 35-40$ in our pooled analysis demonstrates that surgical decompression for SO-MCAi with threatened oedema results in large reductions in mortality (figure 4). Our results emphasise that most of the additional survivors will be left with what many, perhaps most individuals, would consider severe disability-unable to ambulate and needing help with basic needs (potentially all bodily needs), though the proportion with severe versus very severe disability is uncertain (low-quality evidence, table 3 , table 1 and figure $3 \mathrm{~A}$ ). The increase in the proportion of patients left with mild to moderate disability is small and uncertain (low/moderate quality evidence, table 3). Subgroup analyses failed to provide convincing evidence that the impact of mortality differs depending on the timing of surgery or the age of the patient.

\section{Strengths and limitations}

Strengths of our study include explicit eligibility criteria, a comprehensive search, inclusion of all randomised trial data, ${ }^{17} 35-40$ rigorous assessment of risk of bias and reproducible duplicate assessment of both eligibility and risk of bias. We conducted appropriate sensitivity and subgroup analyses and, in addition, rated the quality of evidence using the GRADE approach, ${ }^{28-31}$ a particular contribution of our work.

More specifically, GRADE is a system ${ }^{28-31}$ for rating not individual studies, but rather bodies of evidence addressing the impact of interventions on patientimportant outcomes. In the GRADE system, evidence based on a number of randomised trials begins as high quality, but can be rated down according to any of the five categories of limitations. If individual studies have failed to conceal randomisation, to blind key personnel (in this case outcome assessors) or have stopped early for benefit (all problems in some studies in this review) the body of evidence may be rated down for risk of bias (as we have carried out for functional outcomes in this 
A

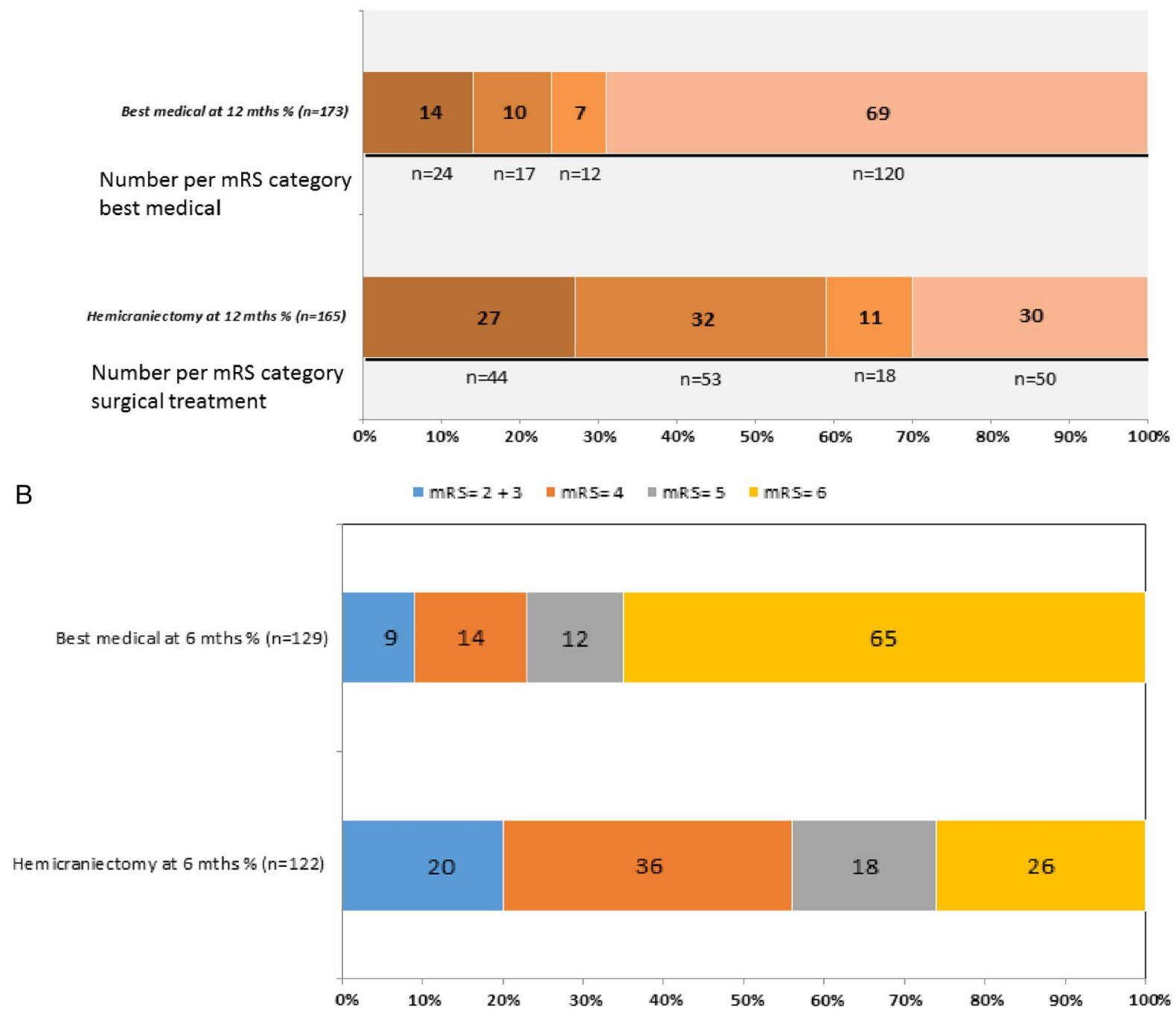

Figure 3 (A): Functional outcome after hemicraniectomy and after medical (conservative) treatment according to the modified Rankin Scale score. (B): Functional outcome after hemicraniectomy and after medical (conservative) treatment according to the modified Rankin Scale score (6 months data, five trials).

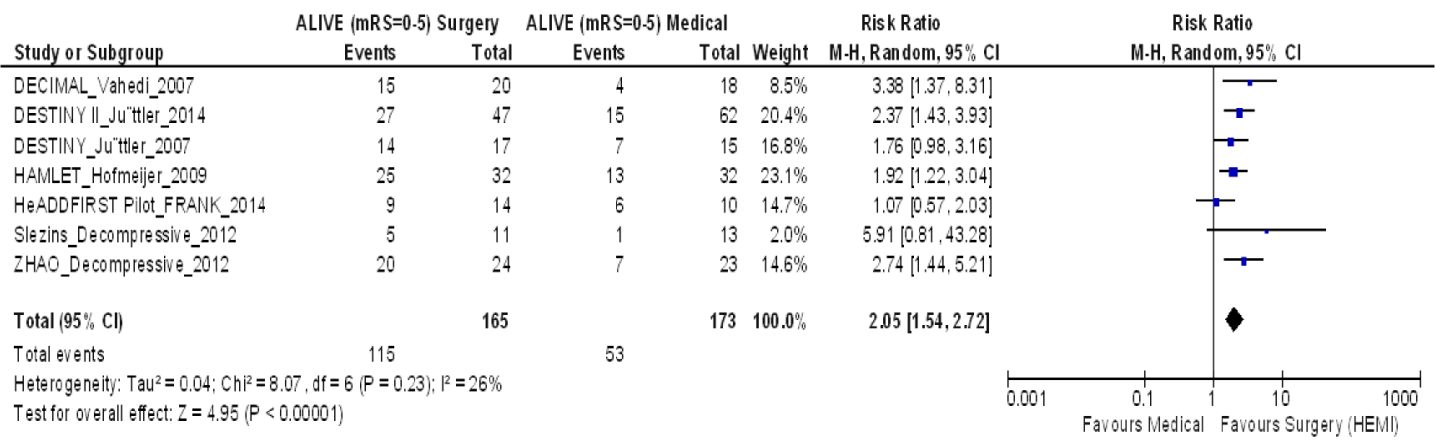

Figure 4 Forest Plot Alive (mRS 0-5) versus Death $(m R S=6)$ at 12 months. mRS, modified Rankin Scale.

review). If sample sizes and number of events are small, and CIs are very wide, quality may be rated down for imprecision (as was the case for functional outcomes in these studies). Other limitations include indirectness (eg, the population enrolled differs from the population of interest), inconsistency (widely divergent results across studies) and publication bias (none of which proved concerns in this review).

An additional strength is the presentation of the numbers/frequencies and percentages by $\mathrm{mRS}$ cut-off point in figures $3 \mathrm{~A}, \mathrm{~B}$ as a means to aid clinicians, surgeons, patients, caregivers and all those involved with 
Table 3 GRADE evidence profile

Patients: aged 18 years and above suffering massive MCA

Intervention: decompressive hemicraniectomy surgery

Comparator: best (standard) medical management

Outcome: death and/or disability at $\mathbf{1 2}$ months follow-up based on mRS scores

Quality assessment

Summary of findings

\section{mRS cut-off}

point; $\boldsymbol{n}$ of

studies

Design

Risk of

$\begin{array}{lll}\text { Consis-tency Direct-ness } & \text { Preci-sion } & \begin{array}{l}\text { Publication } \\ \text { bias }\end{array}\end{array}$

Number of patients

(death); $\mathrm{n}=7$

Randomised $\mathrm{No}$

trials*

No No serious No serious No serious None

None

$\begin{array}{lll}\begin{array}{l}\text { Hemi-craniectomy } \\ \text { surgery }\end{array} & \begin{array}{l}\text { Medical } \\ \text { care }\end{array} & \begin{array}{l}\text { Relative } \\ (95 \% \mathrm{Cl})\end{array}\end{array}$

Absolute effect

surgery

care

(95\% Cl)

Hemi

Quality/certainty

(death); n=7 trials*

Serious
risk of

$\mathrm{n}=165$

Cl)

697 per $1000 \quad 307 \quad$ HIGH

bias

to 2.72 )

390 more per per CONFIDENCE/

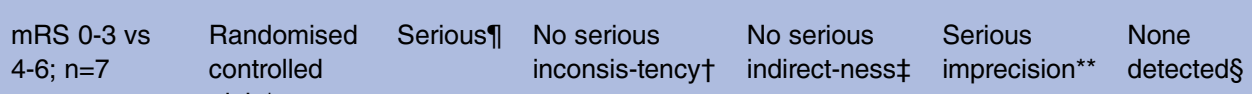

trials*

$\mathrm{n}=165$

$=173$

RR $(95 \%$

Cl)

$1.58(1.02$

1000 patients; 1000 CERTAINTY

$95 \% \mathrm{Cl}$ from

165 to 527

(1)

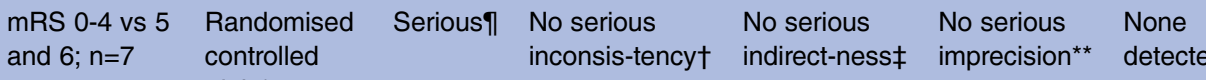

$n=165$

to 2.46$)$

139 LOW

128 more per per CONFIDENCE/

$95 \% \mathrm{Cl}$ from

3 more to 203

more trials*

RR $(95 \% \quad 588$ per 1000

Cl) 351 more 237 MODERATE

$2.25(1.51 \quad 1000$ patients; 1000 CERTAINTY

to 3.35$) \quad 95 \% \mathrm{Cl}$ from

121 more to

557 more

*Six trials that reported complete 12-month follow-up mRS data and one trial based on 6-month follow-up data from the pooled analysis; note while we judged low risk of bias, the reporting of sequence generation could be substantially improved.

†Statistical consistency (heterogeneity): $\chi^{2}$ tests were not significant and $\mathrm{I}^{2} \mathrm{~s}$ were generally low $(<50 \%)$

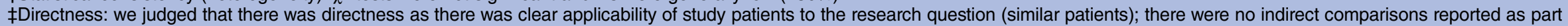
of the included trials.

$\S B$ ased on our exhaustive literature search and the absence of problems of industry funding, we judged that the risk of important publication bias was low.

qWe rated down for risk of bias because in four studies allocation was not concealed, in three studies outcome assessors were not blind to allocation and all but two studies stopped early for

benefit. We did not rate down for the outcome of mortality because it is not subject to bias in outcome assessment.

${ }^{* *}$ Precision: we rated down particularly due to imprecision of estimates as a result of total small sample size and small number of events (particular imprecision was for mRS 0-3).

MCA, middle cerebral artery infarction; $\mathrm{mRS}$, modified Rankin Scale; RR, risk ratio. 


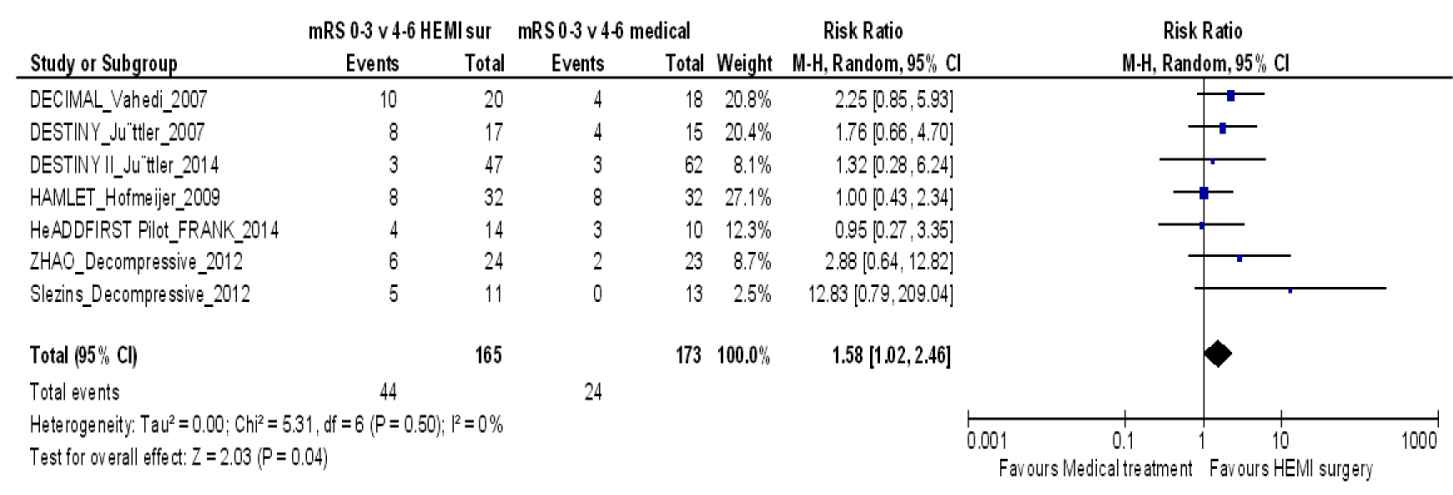

Figure 5 Forest Plot mRS=0-3 vs 4-6, surgery versus medical treatment at 12 months. mRS, modified Rankin Scale.

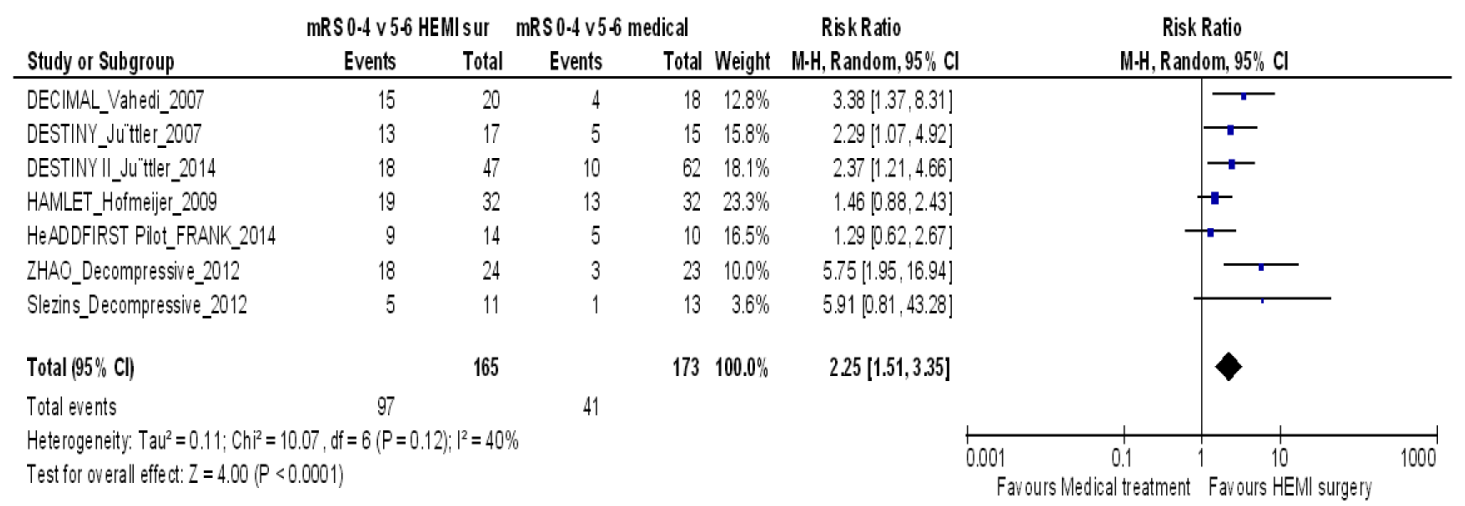

Figure 6 Forest Plot mRS=0-4 vs 5 and 6, surgery versus medical treatment at 12 months. mRS, modified Rankin Scale.

treatment and care decisions presurgeryand postsurgery. Indirect evidence to support such a pictorial representation comes from studies of optimal formats for presenting information to patients and families in the setting of shared decision-making. ${ }^{42} 43$

Limitations of our review are those of the primary studies. Risks of bias problems as mentioned include lack of concealment of randomisation, lack of blinding of outcome assessors and stopping early because of large effects (figure 2 and table 3). Sample sizes were small, and the number of events in those with mild to moderate disability was particularly small (44 in surgery arm and 24 in medical intervention arm).

The use of the mRS as the sole measure of patients' status after stroke represents another limitation. ${ }^{24} 25$ Limitations of the instrument include the subjective judgement required in making the rating without detailed guidance, and its failure to address the subjective experience (quality of life) of the stroke survivors.

\section{Relation to prior work}

Our results are largely consistent with those of other recent reviews ${ }^{22} 23$ of randomised trials of hemicraniectomy after MCA stroke. None of the prior reviews, however, have included all seven randomised controlled studies that contributed to our meta-analysis. Moreover, other reviews did not highlight the limitations associated with risk of bias and stopping early on the basis of results, nor did they apply the GRADE approach that highlights limitations in the evidence. These limitations include both risk of bias and limited sample size and number of events, particularly in the number of patients without severe disability (table 3 ).

One prior study is of note in that it addressed the cost implications of the trial results. Hofmeijer $e t a l^{44}$ assessed clinical outcomes, costs and cost-effectiveness for the first 3 years in patients who were randomised to surgical decompression or best medical treatment using the HAMLET $^{39}$ data. Results suggested that hemicraniectomy increases quality-adjusted life years (QALYs). The health gain comes, however, at large financial costs $(€ 127000$ per QALY gained during the initial 3 years postsurgery with an estimated $€ 60000$ per QALY gained during the patient's lifetime). The Geurts et $a l^{41}$ follow-up study has also provided preliminary indications that the impact of surgery are maintained at 3 years post stroke, based on their re-examination of the HAMLET trial $^{39}$ data.

Prior cohort studies ${ }^{45-48}$ raised the issue of optimal age limits for surgery. We however, found no evidence to suggest a different impact on mortality in those over and under 60 years.

\section{Implications}

Although hemicraniectory reduces mortality, the majority of survivors face a life of severe disability associated 
with large caregiver burden. We have sought to highlight the latter implication of surgery given the challenges this presents to patients and caregivers.

A recent 2014 scientific statement regarding managing patients with a swollen ischaemic stroke in a cerebral or cerebellar hemisphere underscores this critical condition with potentially extensive disability, and the need for immediate, specialised neurointensive care with likely neurosurgical intervention. ${ }^{49}$ The American Heart Association/American Stroke Association guideline ${ }^{49}$ suggests that in patients with supratentorial hemispheric ischaemic stroke, decompressive craniectomy with dural expansion be the course of action in persons who exhibit continual deterioration neurologically. The guideline ${ }^{49}$ while noting that some patients will benefit from the surgery (including those who are disabled but functionally independent), warns that a large proportion of patients who receive decompressive surgery will be significantly disabled with complete dependence on care.

A Statement for Healthcare Professionals from the Neurocritical Care Society and the German Society for Neuro-Intensive Care and Emergency Medicine (evidence-based guidelines for the Management of Large Hemispheric Infarction), ${ }^{50}$ also highlights the extensive disability that many patients undergoing decompressive craniectomy would confront. Their guideline notes the risks due to anaesthesia, surgical risks and the accompanying pain, infection, bleeding, headaches, seizures, neurological deficits and hydrocephalus. ${ }^{50}$ The guideline also points out the financial costs of surgery and subsequent care. ${ }^{50}$ Despite these warnings, the guideline, ${ }^{50}$ which uses GRADE methods ${ }^{28-31}$ recommends (1) decompressive hemicraniectomy after hemispheric infarct (strong recommendation, high quality of evidence), (2) for older patients ( $>60$ years of age), a greater reliance on patient and family input (strong recommendation, moderate quality of evidence) and (3) performing decompressive hemicraniectomy within 24-48 hours of symptom onset and prior to any herniation symptoms (strong recommendation, moderate quality of evidence).

With the prospect for significant disability and thus extensive need for care, decisions regarding hemicraniectomy are therefore high value and preference dependent. Thus, clinicians with access to hemicraniectomy will need to engage in shared decisionmaking and counselling with families/caregivers of patients who have experienced devastating SO-MCAi and are at risk of death from herniation. The decisions are challenging and will be particularly dependent on attitudes toward living in the health state represented by mRS 4 -the largest group of survivors (figure 3A) - that involves being unable to ambulate and dependent on others for at least some bodily needs. The quality of life of caregivers is also an area post-stroke and surgery that has been neglected in the published literature.

\section{CONCLUSION}

Although there is a large mortality reduction with hemicraniectomy in patients with SO-MCAi, the disabled life that faces the survivors and the uncertain magnitude of the increase in the likelihood of surviving with small or moderate disability, will require family members/caregivers to seriously consider the values and preferences of the afflicted patient in deciding whether to proceed with surgery.

\section{Author affiliations}

${ }^{1}$ Department of Clinical Epidemiology and Biostatistics, Health Research Methods, McMaster University, Hamilton, Ontario, Canada

${ }^{2}$ Department of Clinical Epidemiology and Biostatistics, McMaster University, Hamilton, Ontario, Canada

${ }^{3}$ Department of Medicine, University of Toronto, Ontario, Ontario, Canada ${ }^{4}$ Medical Librarian, Health Sciences Library, McMaster University, Hamilton, Ontario, Canada

${ }^{5}$ Centre for Evidence-Based Chinese Medicine, Beijing University of Chinese Medicine, Beijing, China

${ }^{6}$ Department of Family and Community Medicine, Schwartz/Reisman Emergency Medicine Institute, University of Toronto, Toronto, Ontario, Canada ${ }^{7}$ Department of Neurology, All India Institute of Medical Sciences, New Delhi, India

Acknowledgements The authors wish to express their thanks to Ms Supriya Rave of Toronto, Canada, for her help in screening and abstraction of studies/ data. The opinions and interpretations are not to be ascribed to her in any manner, for example, the interpretation/discussion.

Contributors PA took part in study concept and design, acquisition of data, analysis, writing (bulk of research work). DH-A was involved in study design, interpretation, statistical input, interpretation. RS was involved in design, editing, content analysis/interpretation. NB took part in design, search strategy, literature searching and editing. YC, YF, YZ were involved in screening, abstraction, risk of bias assessment, editing. SM was involved in initial study designand editing of various drafts. KP was involved in cosupervision, final editing/critical revisions, interpretation/important intellectual content. GG was involved in supervision, final editing/critical revisions, interpretation/important intellectual content (bulk of oversight). Rave provided initial screening of titles and abstracts and full texts but has no role in the design, analysis, writing or interpretation of this paper.

Funding There is no funding provided for this research project in any manner. All involved persons provided time and expertise and did not act on behalf of any agency or finding body or received any monies in any format, for this work.

Competing interests PA is a recent doctoral student graduate and is an assistant professor at McMaster University. He sits on no boards, receives or received no royalties, no stock options. Family members are not connected to academia and also receive no financial or non-financial payments related to this study as well as not related. He is involved in GRADE methods and a member of the GRADE methods working group. The use of GRADE in this study was to rate the certainty of the estimates of effect and not advocate for the use of GRADE. DH-A is a recent doctoral student graduate and is an assistant professor at McMaster University. She sits on no boards, receives or received no royalties, no stock options. Her role is that of statistical analyst at McMaster University. She is a member of the CLARITY statistical group that provides statistical advice on analysis issues to McMaster researchers. RS is a medical student at the University of Toronto as well as student at McMaster University. He sits on no boards, receives or received no royalties, no stock options. NB is the medical librarian at McMaster University and sits on no boards, receives or received no royalties, no stock options. YC is a current doctoral student at McMaster University. She sits on no boards, receives or received no royalties, no stock options in any manner. YF is a visiting scholar from Beijing, China. She sits on no boards, receives or received no royalties, no stock options in any manner. YZ has recently graduated with a doctorate 
from McMaster University. She sits on no boards, receives or received no royalties, no stock options in any manner. Family members are not connected to academia or this study and also receive no financial or non-financial payments related to this study as well as not related. She is involved in GRADE methods and a member of the GRADE methods working group. SM is a current doctoral student at McMaster and lectures at the University of Toronto in clinical epidemiology. She sits on no boards, receives or received no royalties, no stock options. She also works at the Schwartz/Reisman Emergency Medicine Institute at the University of Toronto as a manager. KP is a Professor of Neurology at the All India Institute of Medical Sciences. New Delhi, India. He sits on no boards, receives or received no royalties, no stock options. GG is a Professor of medicine at McMaster University. He is the founder of GRADE methods used in guideline development and is a member of the Cochrane Collaboration. He is the founder (with Dr David Sackett) of evidence-based medicine. He functions as editor for several journals and sits on several scientific advisory boards. He receives or received no royalties, no stock options. Family member (wife) is connected to academia as lecturer and received no financial or non-financial payments related to this study.

Provenance and peer review Not commissioned; externally peer reviewed.

Data sharing statement The data used to conduct this study are secondarily held data available to the public in peer reviewed journals. As such we do not own the data and have access to the data publicly. We therefore needed no special permissions to use the data and wish to inform BMJ Open that all data used in this document are publicly held.

Open Access This is an Open Access article distributed in accordance with the Creative Commons Attribution Non Commercial (CC BY-NC 4.0) license, which permits others to distribute, remix, adapt, build upon this work noncommercially, and license their derivative works on different terms, provided the original work is properly cited and the use is non-commercial. See: http:// creativecommons.org/licenses/by-nc/4.0/

\section{REFERENCES}

1. Rödén-Jüllig Å. Progressing stroke: epidemiology. Cerebrovasc Dis 1997;7(Suppl 5):2-5.

2. Tei $\mathrm{H}$, Uchiyama $\mathrm{S}$, Ohara $\mathrm{K}$, et al. Deteriorating ischemic stroke in 4 clinical categories classified by the Oxfordshire Community Stroke Project. Stroke 2000;31:2049-54.

3. Castillo J. Deteriorating stroke: diagnostic criteria, predictors, mechanisms and treatment. Cerebrovasc Dis 1999;9(Suppl 3):1-8.

4. Helleberg $\mathrm{BH}$, Ellekjær $\mathrm{H}$, Rohweder $\mathrm{G}$, et al. Mechanisms, predictors and clinical impact of early neurological deterioration: the protocol of the Trondheim early neurological deterioration study. BMC Neurol 2014;14:201.

5. Bamford J, Sandercock P, Dennis M, et al. Classification and natural history of clinically identifiable subtypes of cerebral infarction. Lancet 1991;337:1521-6

6. Saito I, Segawa H, Shiokawa $Y$, et al. Middle cerebral artery occlusion: correlation of computed tomography and angiography with clinical outcome. Stroke 1987;18:863-8.

7. Aiyagari V, Diringer MN. Management of large hemispheric strokes in the neurological intensive care unit. Neurologist 2002;8:152-62.

8. Vahedi K, Hofmeijer J, Juettler E, et al., DECIMAL, DESTINY, and HAMLET investigators. Early decompressive surgery in malignant infarction of the middle cerebral artery: a pooled analysis of three randomised controlled trials. Lancet Neurol 2007;6:215-22.

9. Kasner SE, Demchuk AM, Berrouschot J, et al. Predictors of fatal brain oedema in massive hemispheric ischemic stroke. Stroke 2001;32:2117-23.

10. Hacke W, Schwab S, Horn M, et al. 'Malignant' middle cerebra artery territory infarction: clinical course and prognostic signs. Arch Neurol 1996;53:309-15.

11. Chu SY, Sheth KN. Decompressive craniectomy in neurocritical care. Curr Treat Options Neurol 2015;17:330.

12. Kim H, Jin ST, Kim YW, et al. Predictors of malignant brain oedema in middle cerebral artery infarction observed on CT angiography. J Clin Neurosci 2015;22:554-60.

13. Schwab S, Aschoff A, Spranger M, et al. The value of intracranial pressure monitoring in acute hemispheric stroke. Neurology 1996;47:393-8.

14. Hofmeijer J, van der Worp HB, Kappelle LJ. Treatment of space-occupying cerebral infarction. Crit Care Med 2003;31:617-25.
15. Huttner HB, Schwab S. Malignant middle cerebral artery infarction: clinical characteristics, treatment strategies, and future perspectives. Lancet Neurol 2009;8:949-58.

16. Wang DZ, Nair DS, Talkad AV. Acute decompressive hemicraniectomy to control high intracranial pressure in patients with malignant MCA ischemic strokes. Curr Treat Options Cardiovasc Med 2011;13:225-32.

17. Jüttler E, Unterberg A, Woitzik J, et al., DESTINY II Investigators. Hemicraniectomy in older patients with extensive middle-cerebralartery stroke. N Engl J Med 2014;370:1091-100.

18. Creutzfeldt CJ, Schubert GB, Tirschwell DL, et al. Abstract 155: risk of Seizures after malignant MCA stroke and decompressive hemicraniectomy. International Stroke Conference Oral Abstracts. Stroke 2012;43:A155

19. Wagner $\mathrm{S}$, Schnippering $\mathrm{H}$, Aschoff $\mathrm{A}$, et al. Suboptimum hemicraniectomy as a cause of additional cerebral lesions in patients with malignant infarction of the middle cerebral artery. J Neurosurg 2001;94:693-6.

20. Sarov M, Guichard JP, Chibarro S, et al. DECIMAL investigators. Sinking skin flap syndrome and paradoxical herniation after hemicraniectomy for malignant hemispheric infarction. Stroke 2010;41:560-2.

21. Cruz-Flores S, Berge E, Whittle IR. Surgical decompression for cerebral oedema in acute ischaemic stroke. Cochrane Database Syst Rev 2012;1:CD003435.

22. Back L, Nagaraja V, Kapur A, et al. The role of decompressive hemicraniectomy in extensive middle cerebral artery STROKES: a meta-analysis of randomized trials. Intern Med J 2015;45:711-17.

23. Yang $\mathrm{MH}$, Lin HY, Fu J, et al. Decompressive hemicraniectomy in patients with malignant middle cerebral artery infarction: a systematic review and meta-analysis. Surgeon 2015;13:230-40.

24. Rankin J. Cerebral vascular accidents in patients over the age of 60 . II. Prognosis. Scott Med J 1957;2:200-15.

25. Bonita R, Beaglehole R. Recovery of motor function after stroke. Stroke 1988;19:1497-500.

26. 8.5 The Cochrane Collaboration's tool for assessing risk of bias. http:// handbook.cochrane.org/chapter $8 / 85$ the cochrane collaborations tool for assessing risk of bias.htm (accessed 27 Jan 2015).

27. Guyatt $\bar{G}$, Busse J. Methods commentary: risk of bias in randomized

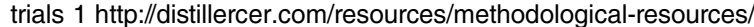
risk-of-bias-commentary/ (accessed 31 Jan 2015).

28. Guyatt $\mathrm{GH}$, Oxman AD, Vist GE, et al. GRADE Working Group. GRADE: an emerging consensus on rating quality of evidence and strength of recommendations. BMJ 2008;336:924-6.

29. Balshem H, Helfand $M$, Schünemann HJ, et al. GRADE guidelines: 3 . Rating the quality of evidence. J Clin Epidemio 2011;64:401-6

30. Andrews JC, Schünemann HJ, Oxman AD, et al. GRADE guidelines 15: going from evidence to recommendation-determinants of a recommendation's direction and strength. J Clin Epidemiol 2013;66:726-35.

31. Guyatt G, Oxman AD, Akl EA, et al. GRADE guidelines: 1. Introduction-GRADE evidence profiles and summary of findings tables. J Clin Epidemiol 2011;64:383-94.

32. Akl EA, Johnston BC, Alonso-Coello P, et al. Addressing dichotomous data for participants excluded from trial analysis: a guide for systematic reviewers. PLOS ONE 2013;8:e57132.

33. Landis JR, Koch GG. The measurement of observer agreement for categorical data. Biometrics 1977;33:159-74.

34. RevMan 5. http://tech.cochrane.org/revman/download (accessed 31 Jan 2015).

35. Frank JI, Schumm LP, Wroblewski K, et al, HeADDFIRST Trialists. Hemicraniectomy and durotomy on deterioration from infarctionrelated swelling trial: randomized pilot clinical trial. Stroke 2014;45:781-7.

36. Zhao J, Su YY, Zhang Y, et al. Decompressive hemicraniectomy in malignant middle cerebral artery infarct: a randomized controlled trial enrolling patients up to 80 years old. Neurocrit Care 2012;17: 161-71.

37. Vahedi K, Vicaut E, Mateo J, et al., DECIMAL investigators. Sequential design, multicenter, randomized, controlled trial of early decompressive craniectomy in malignant middle cerebral artery infarction (DECIMAL Trial). Stroke 2007;38:2506-17.

38. Jüttler E, Schwab S, Schmiedek P, et al., DESTINY Study Group. Decompressive surgery for the treatment of malignant infarction of the middle cerebral artery (DESTINY): a randomized, controlled trial. Stroke 2007;38:2518-25

39. Hofmeijer J, Kappelle LJ, Algra A, et al., HAMLET investigators. Surgical decompression for space-occupying cerebral infarction (the Hemicraniectomy After Middle Cerebral Artery infarction with Life-threatening Edema Trial [HAMLET]): a multicentre, open, randomised trial. Lancet Neurol 2009;8:326-33. 
40. Slezins $\mathrm{J}$, Keris $\mathrm{V}$, Bricis $\mathrm{R}$, et al. Preliminary results of randomized controlled study on decompressive craniectomy in treatment of malignant middle cerebral artery stroke. Medicina (Kaunas). 2012;48:521-4

41. Geurts M, van derWorp HB, Kappelle LJ, et al. Surgical decompression for space-occupying cerebral infarction: outcomes at 3 years in the randomized HAMLET trial. Stroke 2013;44:2506-8

42. Epstein RM, Alper BS, Quill TE. Communicating evidence for participatory decision-making. JAMA 2004;291:2359-66.

43. Woloshin S, Schwartz LM. Communicating data about the benefits and harms of treatment: a randomized trial. Ann Intern Med 2011;155:87-96.

44. Hofmeijer J, van der Worp HB, Kappelle LJ, et al. HAMLET Steering Committee. Cost-effectiveness of surgical decompression for space-occupying hemispheric infarction. Stroke 2013;44:2923-5.

45. Arac A, Blanchard V, Lee M, et al. Assessment of outcome following decompressive craniectomy for malignant middle cerebral artery infarction in patients older than 60 years of age. Neurosurg Focus 2009;26:E3.
46. Holtkamp M, Buchheim K, Unterberg A, et al. Hemicraniectomy in elderly patients with space occupying media infarction: improved survival but poor functional outcome. J Neurol Neurosurg Psychiatry 2001;70:226-8

47. Yao $\mathrm{Y}$, Liu $\mathrm{W}$, Yang $\mathrm{X}$, et al. Is decompressive craniectomy for malignant middle cerebral artery territory infarction of any benefit for elderly patients? Surg Neurol 2005;64:165-9.

48. van der Worp HB, Kappelle LJ. Early decompressive hemicraniectomy in older patients with nondominant hemispheric infarction does not improve outcome. Stroke 2011;42:845-6.

49. Wijdicks EF, Sheth KN, Carter BS, et al., American Heart Association Stroke Council. Recommendations for the management of cerebral and cerebellar infarction with swelling: a statement for healthcare professionals from the American Heart Association/American Stroke Association. Stroke 2014;45:1222-38.

50. Torbey MT, Bösel J, Rhoney DH, et al. Evidence-based guidelines for the management of large hemispheric infarction : a statement for healthcare professionals from the Neurocritical Care Society and the German Society for Neuro-intensive Care and Emergency Medicine. Neurocrit Care 2015;22:146-64. 